\title{
LOG-CONVEXITY OF COMBINATORIAL SEQUENCES FROM THEIR CONVEXITY
}

\author{
TOMISLAV DošLIĆ
}

Abstract. A sequence $\left(x_{n}\right)_{n \geqslant 0}$ of positive real numbers is log-convex if the inequality $x_{n}^{2} \leqslant$ $x_{n-1} x_{n+1}$ is valid for all $n \geqslant 1$. We show here how the problem of establishing the log-convexity of a given combinatorial sequence can be reduced to examining the ordinary convexity of related sequences. The new method is then used to prove that the sequence of Motzkin numbers is log-convex.

Mathematics subject classification (2000): 05A20, 05A16, $11 \mathrm{~B} 37$.

Keywords and phrases: Log-convexity, convexity, combinatorial sequences, Motzkin numbers.

\section{REFERENCES}

[1] M. Aigner, Motzkin numbers, European J. Combin., 19 (1998), 663-675.

[2] D. V. Anosov, On the sum of log-convex functions, Math. Prosv., 5 (2002), 158-163 (in Russian).

[3] N. Asai, I. Kubo, H.-H. Kuo, Bell numbers, log-concavity, and log-convexity, Acta Appl. Math., 63 (2000), 79-87.

[4] E. A. BENDER AND E. R. CANFIELD, Log-concavity and related properties of the cycle index polynomials, J. Combin. Theory A, 74 (1996), 56-70.

[5] M. BónA, R. EHRENBORG, A combinatorial proof of the log-concavity of the numbers of permutations with $k$ runs, J. Combin. Theory A, 90 (2000), 293-303.

[6] F. Brenti, Unimodal, log-concave and Pólya frequency sequences in combinatorics, American Math. Society, Providence, RI, 1989.

[7] D. CALLAN, Notes on Motzkin and Schröder numbers, preprint, 2000.

[8] T. DošLić, Log-balanced combinatorial sequences, Int. J. Math. Math. Sci., 2005 (2005), 507-522.

[9] T. DošLić, D. SVRTAN AND D. VelJAN, Enumerative aspects of secondary structures, Discrete Math., 285 (2004), 67-82.

[10] T. DošLić AND D. Veljan, Calculus proofs of some combinatorial inequalities, Math. Inequal. Appl., 6 (2003), 197-209.

[11] T. DošLiĆ AND D. Veljan, Logarithmic behavior of some combinatorial sequences, Discrete Math., 308 (2008), 2182-2212.

[12] L. L. LiU, Y. WANG, On the log-convexity of combinatorial sequences, Adv. Appl. Math., 39 (2007), 453-476.

[13] P. Montel, Sur les functions convexes et les fonctions sousharmoniques, J. Math. Pures et Appl., 7 (1928), 29-60.

[14] B. SaGAN, Inductive and injective proofs of log-concavity results, Discrete Math., 68 (1988), 281292.

[15] R. P. StANLEY, Log-concave and unimodal sequences in algebra, combinatorics and geometry, Ann. N.Y. Acad. Sci., 576 (1989), 500-535.

[16] R. P. Stanley, Positivity problems and conjectures in algebraic combinatorics, in Mathematics: Frontiers and Perspectives, Eds. V. Arnold et al., IMU-AMS, 2000, 295-319.

[17] R. P. Stanley, Enumerative Combinatorics vol. 2, Cambridge Univ. Press, Cambridge, 1999.

[18] Y. WANG, Linear transformations preserving log-concavity, Lin. Alg. Appl., 359 (2003), 161-167. 